\title{
EMBEDDINGS OF MÜNTZ SPACES: THE HILBERTIAN CASE
}

\author{
S. WALEED NOOR AND DAN TIMOTIN
}

(Communicated by Richard Rochberg)

\begin{abstract}
Given a strictly increasing sequence $\Lambda=\left(\lambda_{n}\right)$ of nonnegative real numbers, with $\sum_{n=1}^{\infty} \frac{1}{\lambda_{n}}<\infty$, the Müntz spaces $M_{\Lambda}^{p}$ are defined as the closure in $L^{p}([0,1])$ of the monomials $x^{\lambda_{n}}$. We discuss properties of the embedding $M_{\Lambda}^{p} \subset L^{p}(\mu)$, where $\mu$ is a finite positive Borel measure on the interval $[0,1]$. Most of the results are obtained for the Hilbertian case $p=2$, in which we give conditions for the embedding to be bounded, compact, or to belong to the Schatten-von Neumann ideals.
\end{abstract}

\section{INTRODUCTION}

The Müntz-Szasz Theorem states that if $0=\lambda_{0}<\lambda_{1}<\cdots<\lambda_{n}<\ldots$ is an increasing sequence of nonnegative real numbers, then the linear span of $x^{\lambda_{n}}$ is dense in $C([0,1])$ if and only if $\sum_{n=1}^{\infty} \frac{1}{\lambda_{n}}=\infty$. When $\sum_{n=1}^{\infty} \frac{1}{\lambda_{n}}<\infty$, the closed linear span of the monomials $x^{\lambda_{n}}$ in different Banach spaces that contain them is usually not equal to the whole space. In particular, if $1 \leq p<+\infty$, the closed linear span of the monomials $x^{\lambda_{n}}, n \geq 0$, in $L^{p}([0,1])$ is a proper subspace of $L^{p}([0,1])$. These spaces, called Müntz spaces and denoted $M_{\Lambda}^{p}$, exhibit interesting properties that have not been very much investigated. We refer principally to the monographs 4, 9]; recent results appear in [1, 2, 11, 6.

Our starting point is the paper [6], which tries to find conditions under which the space $M_{\Lambda}^{1}$ is continuously embedded in the Lebesgue space $L^{1}(\mu)$. In full generality the problem is rather difficult; more precise results are obtained for special classes of sequences $\Lambda$ and/or measures $\mu$. One can see therein that, as a general rule, the embedding properties are related to the behavior of $\mu$ near the point 1 , which for these problems acts as a kind of "distinguished boundary" of the unit interval.

The purpose of this paper is to investigate embedding results for other Müntz spaces; we will actually focus on the Hilbert space $M_{\Lambda}^{2}$, although occasionally other values of $p$ also enter the picture. In the case of $M_{\Lambda}^{2}$ we will also treat more refined properties of the embedding, namely its possible belonging to Schatten-von Neumann classes. As expected, the behavior of $\mu$ near the point 1 is again decisive. Examples are given to illustrate the difficulties.

The plan of the paper is the following. The next section contains necessary preliminaries. In Section 3 we associate to a sequence $\Lambda$ a certain real-valued function $\psi$; this function allows us to formulate in a unitary manner some general

Received by the editors September 18, 2011.

2010 Mathematics Subject Classification. Primary 46E15, 46E20, 46 E35.

Key words and phrases. Müntz space, embedding measure, lacunary sequence, Schatten-von Neumann classes. 
embedding results. Sections 4 and 6 focus on particular classes of measures and sequences, while in Section 5 an interpolation result of Riesz-Thorin type is proved for embedding operators. Finally, Section 7 presents two interesting examples.

\section{Preliminaries}

2.1. Riesz sequences and bases. A sequence $\left\{f_{k}\right\}_{k=1}^{\infty}$ in a Hilbert space $H$ is said to be complete if $\operatorname{span}\left\{f_{k}\right\}_{k \geq 1}$ is dense in $H$. A Riesz sequence for a separable Hilbert space $H$ is a sequence $\left\{f_{k}\right\}_{k=1}^{\infty}$ such that there exists a constant $C>0$ for which

$$
\frac{1}{C} \sum_{k=1}^{m}\left|c_{k}\right|^{2} \leq\left\|\sum_{k=1}^{m} c_{k} f_{k}\right\|^{2} \leq C \sum_{k=1}^{m}\left|c_{k}\right|^{2}
$$

for every finite scalar sequence $\left\{c_{k}\right\}_{k=1}^{\infty}$. A Riesz basis is a complete Riesz sequence.

The following proposition gathers some well-known facts about Riesz sequences (see, for instance, [7]).

Lemma 2.1. Suppose $\left(x_{n}\right)$ is a sequence of vectors in the Hilbert space $\mathcal{F}$, and define its Gramian to be the infinite matrix $\Gamma=\left(\left\langle x_{n}, x_{m}\right\rangle\right)_{n, m \in \mathbb{N}}$. Then:

(i) $\left(x_{n}\right)$ is a Riesz sequence if and only if $\Gamma$ defines an invertible operator on $\ell^{2}(\mathbb{N})$.

(ii) If $\left(g_{n}\right)_{n \in \mathbb{N}}$ is a Riesz basis in the Hilbert space $\mathcal{E}$, then $g_{n} \mapsto x_{n}$ can be extended to a bounded linear operator $J: \mathcal{E} \rightarrow \mathcal{F}$ if and only if $\Gamma$ defines a bounded operator on $\ell^{2}(\mathbb{N})$; we have $\|J\|=\|\Gamma\|^{1 / 2}$.

(iii) The sequence $\left(x_{n}\right)$ is a Riesz basis if and only if, for some (equivalently, any) Riesz basis $\left(g_{n}\right)_{n \in \mathbb{N}}$ in the Hilbert space $\mathcal{E}, g_{n} \mapsto x_{n}$ can be extended to an invertible linear operator $J: \mathcal{E} \rightarrow \mathcal{F}$.

2.2. Schatten-Von Neumann classes. For $0<q<\infty$, the Schatten-Von Neumann class $\mathcal{S}_{q}\left(\mathcal{H}_{1}, \mathcal{H}_{2}\right)$ is formed by the compact Hilbert space operators $T: \mathcal{H}_{1} \rightarrow$ $\mathcal{H}_{2}$ such that $|T|=\sqrt{T^{*} T}: \mathcal{H}_{1} \rightarrow \mathcal{H}_{1}$ has a family of eigenvalues $\left\{s_{n}(T)\right\}_{n=1}^{\infty} \in \ell_{q}$. If we define

$$
\|T\|_{q}=\left(\sum_{n=1}^{\infty} s_{n}(T)^{q}\right)^{1 / q},
$$

then we obtain a quasinorm for $0<q<1$ and a norm for $q \geq 1$, with respect to which $\mathcal{S}_{q}\left(\mathcal{H}_{1}, \mathcal{H}_{2}\right)$ is complete. It is immediate that $\|T\|_{q} \geq\|T\|_{q^{\prime}}$ for $q \leq q^{\prime}$; hence $\mathcal{S}_{q} \subset \mathcal{S}_{q^{\prime}}$. Again we gather in a lemma some properties that we will use; more information can be found, for instance, in [10, 8]. For the sake of this lemma, we will denote by $\|T\|_{\infty}$ the usual operator norm.

Lemma 2.2. Suppose $T^{\prime}: \mathcal{H}_{0} \longrightarrow \mathcal{H}_{1}, T: \mathcal{H}_{1} \longrightarrow \mathcal{H}_{2}$ are bounded operators, $0<q, q^{\prime} \leq \infty$.

(i) If $T \in \mathcal{S}_{q}\left(\mathcal{H}_{1}, \mathcal{H}_{2}\right), T^{\prime} \in \mathcal{S}_{q^{\prime}}\left(\mathcal{H}_{0}, \mathcal{H}_{1}\right)$ and $1 / r=1 / q+1 / q^{\prime}$, then $T^{\prime} T \in \mathcal{S}_{r}$, and

$$
\left\|T T^{\prime}\right\|_{r} \leq\|T\|_{s}\left\|T^{\prime}\right\|_{s^{\prime}}
$$

(ii) If $0<q \leq 2$, then

$$
\|T\|_{\mathcal{S}_{q}}^{q}=\inf \sum_{n}\left\|T \phi_{n}\right\|_{\mathcal{H}_{2}}^{q},
$$

where the infimum is taken over all orthonormal bases $\left(\phi_{n}\right)_{n}$ of $\mathcal{H}_{1}$. 
(iii) If $2 \leq q<\infty$, then

$$
\|T\|_{\mathcal{S}_{q}}^{q}=\sup \sum_{n}\left\|T \phi_{n}\right\|_{\mathcal{H}_{2}}^{q},
$$

where the supremum is taken over all orthonormal bases $\left(\phi_{n}\right)_{n}$ of $\mathcal{H}_{1}$.

Note that the right-hand side in (i) and (ii) may be infinite, meaning that $T \notin \mathcal{S}_{q}$. We will use the following corollary of Lemma 2.1 (iii) and Lemma 2.2 (ii).

Corollary 2.3. Suppose $T: \mathcal{H}_{1} \longrightarrow \mathcal{H}_{2}$ is a bounded operator and $\left(x_{n}\right)$ is a Riesz basis in $\mathcal{H}_{1}$.

(i) If $\frac{T x_{n}}{\left\|T x_{n}\right\|}$ is a Riesz sequence in $\mathcal{H}_{2}$, then, for all $q>0, T \in \mathcal{S}_{q}$ if and only if $\left(\left\|T x_{n}\right\|\right) \in \ell^{q}$.

(ii) If $0<q \leq 2$, then

$$
\|T\|_{\mathcal{S}_{q}}^{q} \leq \sum_{n}\left\|T x_{n}\right\|_{\mathcal{H}_{2}}^{q}
$$

In particular, $T \in \mathcal{S}_{q}$ whenever the right-hand side is finite for some Riesz basis $\left(x_{n}\right)$.

The elements of $\mathcal{S}_{2}\left(\mathcal{H}_{1}, \mathcal{H}_{2}\right)$ are called Hilbert-Schmidt operators and by Lemma 2.2 the Hilbert-Schmidt norm of $T \in \mathcal{S}_{2}\left(\mathcal{H}_{1}, \mathcal{H}_{2}\right)$ is given by

$$
\|T\|_{\mathcal{S}_{2}}=\left(\sum_{n}\left\|T \phi_{n}\right\|_{\mathcal{H}_{2}}^{2}\right)^{1 / 2}
$$

where $\left(\phi_{n}\right)_{n}$ is any orthonormal basis of $\mathcal{H}_{1}$.

2.3. Müntz spaces and embeddings. We denote by $m$ the Lebesgue measure on $[0,1]$ and by $\|\cdot\|_{p}$ the norm in $L^{p}(m)$ for $1 \leq p \leq \infty$.

Suppose $\Lambda=\left\{\lambda_{n}\right\}_{n \geq 1}$ is an increasing sequence of positive real numbers with $\sum_{n=1}^{\infty} \frac{1}{\lambda_{n}}<\infty$. As discussed in the introduction, the Müntz space $M_{\Lambda}^{p}$ is defined to be the closure of the monomials $x^{\lambda_{n}}, n \geq 1$ in $L^{p}(m)$; which is a proper subspace of $L^{p}(m)$ by the Müntz-Szasz Theorem. It is proven in [4, 9] that the functions in $M_{\Lambda}^{p}$ are actually real analytic on the interval $(0,1)$ and continuous on $[0,1)$.

We will use the following two results concerning Müntz spaces that appear in 9 .

Lemma 2.4 ([9], Corollary 8.1.2). Any Müntz polynomial $f(x)=\sum_{k=1}^{m} \alpha_{k} x^{\lambda_{k}}$ satisfies

$$
|f(x)| \leq 2\left(\sum_{k=1}^{m} x^{\lambda_{k} \beta_{k}}\right)\|f\|_{\infty}
$$

for any $x \in[0,1]$ and any $\beta_{k} \geq 0$ with $\sum_{k=1}^{m} \beta_{k}=1$.

Lemma 2.5 ([9], Proposition 8.2.2). There is a constant $K>0$ (depending only on $\Lambda)$ such that, if $f(x)=\sum_{k=1}^{m} \alpha_{k} x^{\lambda_{n}}$, then

$$
\left\|f^{\prime}\right\|_{\infty} \leq K\left(\sum_{k=1}^{m} \lambda_{k}\right)\|f\|_{\infty} .
$$

For a fixed $p \geq 1$, a positive measure $\mu$ on $[0,1]$ is called $\Lambda_{p}$-embedding if there is a constant $C>0$ such that

$$
\|g\|_{L^{p}(\mu)} \leq C\|g\|_{p}
$$


for all polynomials $g \in M_{\Lambda}^{p}$. Whenever $p$ is clear from the context, we will remove the subscript $p$ and use the notation $\Lambda$-embedding.

It follows easily from the definition (see [6]) that a $\Lambda_{p}$-embedding measure $\mu$ has to satisfy $\mu(1)=0$. Therefore, as in Remark 2.5 of [6], we may extend the embedding to all $f \in M_{\Lambda}^{p}$ : if $\mu$ is $\Lambda_{p}$-embedding, then $M_{\Lambda}^{p} \subset L^{p}(\mu)$ and $\|f\|_{L^{p}(\mu)} \leq$ $C\|f\|_{p}$ for all $f \in M_{\Lambda}^{p}$. For a $\Lambda_{p}$-embedding $\mu$ we denote by $i_{\mu}^{p}$ the embedding operator $i_{\mu}^{p}: M_{\Lambda}^{p} \hookrightarrow L^{p}(\mu)$, which is bounded. If $0<\varepsilon<1$, then the interval $[1-\varepsilon, 1]$ will be denoted by $J_{\varepsilon}$.

If $T: \mathcal{E} \rightarrow \mathcal{F}$ is a bounded operator on Banach spaces, we define by $\|T\|_{e}=$ $\inf _{\mathcal{K}}\|T+\mathcal{K}\|$ the essential norm of an operator, where the infimum is taken over all compact operators $\mathcal{K}: \mathcal{E} \rightarrow \mathcal{F}$. This norm measures how far an operator is from being compact. In particular, $T$ is compact if and only if $\|T\|_{e}=0$. If $\mu$ is a positive measure on $[0,1]$, we will denote by $\mu_{m}$ the measure equal to $\mu$ on $\left[0,1-\frac{1}{m}\right]$ and 0 elsewhere, and $\mu_{m}^{\prime}=\mu-\mu_{m}$.

The next proposition gathers some facts that are analogues of the corresponding results obtained in [6] for $p=1$ and can be proved by adapting the methods therein; we state them without further comment.

Proposition 2.6. (i) If for some $\varepsilon>0$ the restriction of $\mu$ to the interval $[1-\varepsilon, 1]$ is absolutely continuous with respect to $\left.m\right|_{[1-\varepsilon, 1]}$, with essentially bounded density, then $\mu$ is $\Lambda$-embedding for any $\Lambda$.

(ii) If $\operatorname{supp} \mu \subset[0,1-\varepsilon]$, then $i_{\mu}$ is compact.

(iii) If $\mu$ is an embedding measure, then

$$
\left\|i_{\mu}\right\|_{e}=\lim _{n \rightarrow \infty}\left\|i_{\mu_{n}^{\prime}}\right\|
$$

(iv) Suppose there exists $\delta>0$ such that $\left.d \mu\right|_{J_{\delta}}=\left.h d m\right|_{J_{\delta}}$ for some bounded measurable function $h$ with $\lim _{t \rightarrow 1} h(t)=a$. Then $i_{\mu}$ is bounded and $\left\|i_{\mu}\right\|_{e}=a^{1 / p}$.

We will have the occasion to use the following elementary lemma.

Lemma $2.7\left([6]\right.$, Lemma 2.2). Suppose $\rho: \mathbb{R}_{+} \longrightarrow \mathbb{R}_{+}$is an increasing, $\mathcal{C}^{1}$ function with $\rho(0)=0$ such that $\mu\left(J_{\varepsilon}\right) \leq \rho(\varepsilon)$ for all $\varepsilon \in(0,1]$. Then for any continuous, positive, increasing function $g$ we have

$$
\int_{[0,1]} g d \mu \leq \int_{0}^{1} g(x) \rho^{\prime}(1-x) d x .
$$

A sequence $\Lambda$ is lacunary if for some $\gamma>1$ we have $\lambda_{n+1} / \lambda_{n} \geq \gamma, n \geq 1$. The main feature of lacunarity is that the monomials $\lambda_{n}^{1 / p} x^{\lambda_{n}}$ form a Riesz basis in each of the spaces $M_{\Lambda}^{p}$. In particular, the sequence $\left(\lambda_{n}^{1 / 2} x^{\lambda_{n}}\right)_{n \geq 1}$ forms a Riesz basis in $M_{\Lambda}^{2}$.

A more general class of sequences is defined as follows. The sequence $\Lambda$ is called quasilacunary if for some increasing sequence $\left\{n_{k}\right\}$ of integers with $N:=$ $\sup _{k}\left(n_{k+1}-n_{k}\right)<\infty$ and some $\gamma>1$ we have $\lambda_{n_{k+1}} / \lambda_{n_{k}} \geq \gamma$. It is easy to show that any quasilacunary sequence may be enlarged to one that is still quasilacunary and satisfies $\lambda_{n+1} / \lambda_{n} \leq \gamma^{2}$. The main property of quasilacunary sequences is contained in the following lemma. 
Lemma 2.8 ([9], Theorem 9.3.3). If $\Lambda$ is quasilacunary and $F_{k}=\operatorname{span}\left\{x^{\lambda_{n_{k}+1}}, \ldots\right.$, $\left.x^{\lambda_{n_{k+1}}}\right\}$, then $\exists d_{1}, d_{2}>0$ such that for any sequence of functions $f_{k} \in F_{k}$ we have

$$
d_{1}\left(\sum_{k}\left\|f_{k}\right\|_{2}^{2}\right) \leq\left\|\sum_{k} f_{k}\right\|_{2}^{2} \leq d_{2}\left(\sum_{k}\left\|f_{k}\right\|_{2}^{2}\right) .
$$

\section{Embeddings in $M_{\Lambda}^{2}$}

Most of our results pertain to the Hilbert space $M_{\Lambda}^{2}$. In particular, in this section we will consider only $p=2$, and therefore we will drop the index $p$ and write " $\Lambda$-embedding" and " $i_{\mu}$ ". On the other hand, we will complicate things slightly by introducing $M_{\Lambda, a}^{2}$ as the closure of the same monomials $x^{\lambda_{n}}$ in $L^{2}([0, a])$; so $M_{\Lambda}^{2}=M_{\Lambda, 1}^{2}$.

It is known (see 4], pp. 177-178) that the condition $\sum_{n} 1 / \lambda_{n}<\infty$ ensures that the system $x^{\lambda_{n}}$ is minimal in $M_{\Lambda}^{2}$, and that, if $d_{n}$ is the distance in $M_{\Lambda}^{2}$ from $x^{\lambda_{n}}$ to the linear space $\hat{\mathcal{P}}_{n}$ spanned by $x^{\lambda_{m}}$ with $m \neq n$, then $d_{n}=e^{-\gamma_{n} \lambda_{n}}$, with $\gamma_{n} \rightarrow 0$. Let us denote

$$
\psi(x)=\sum_{n \geq 1} d_{n}^{-1} x^{\lambda_{n}} .
$$

The remarks above show that the sum is convergent for any $x<1$ and defines an increasing function of $x$.

A simple argument of Hilbert space (also reproduced in 4, pp. 177-178) says that if $p=\sum_{i} \alpha_{i} x^{\lambda_{i}}$, then

$$
\left|\alpha_{n}\right| \leq d_{n}^{-1}\|p\|_{2}
$$

It follows then that, for any $f \in M_{\Lambda}^{2}$, we have the estimate

$$
\left|f^{(k)}(x)\right| \leq \psi^{(k)}(x)\|f\|_{2}, \quad k=0,1, \ldots
$$

$\left(f^{(k)}\right.$ denoting, as usual, the $k$ th derivative of $f$ ).

Now consider $0<a<1$. If $d_{n}(a)$ is the distance in $M_{\Lambda, a}^{2}$ from $x^{\lambda_{n}}$ to $\hat{\mathcal{P}}_{n}$, then

$$
d_{n}(a)^{2}=\inf _{p \in \hat{\mathcal{P}}_{n}} \int_{0}^{a}\left|x^{\lambda_{n}}-p(x)\right|^{2} d x=\inf _{p \in \hat{\mathcal{P}}_{n}} \int_{0}^{1}\left|a^{\lambda_{n}} t^{\lambda_{n}}-p(t)\right|^{2} a d t=a^{2 \lambda_{n}+1} d_{n},
$$

whence

$$
\psi_{a}(x):=\sum_{n \geq 1} d_{n}(a)^{-1} x^{\lambda_{n}}=a^{-1 / 2} \psi\left(a^{-1} x\right) .
$$

We have thus the estimate, for functions in $M_{\Lambda, a}^{2}$,

$$
|f(x)| \leq a^{-1 / 2} \psi\left(a^{-1} x\right)\|f\|_{2} .
$$

In particular, if $a=1$, we recapture (3.2) for $k=0$.

Although the function $\psi$ is a rather rough indicator of the properties of the sequences $\Lambda$, it is useful in obtaining sufficient conditions for embedding results. A first example is an analogue for $M_{\Lambda}^{2}$ of [6, Theorem 2.6].

Theorem 3.1. If $\psi \in L^{2}(\mu)$, then $\mu$ is $\Lambda$-embedding and $\left\|i_{\mu}\right\| \leq\|\psi\|_{L^{2}(\mu)}$.

Proof. The proof consists in integrating with respect to $\mu$ the relation $|f(x)| \leq$ $\psi(x)\|f\|_{2}$, which, as noted above, is the case $k=0$ of (3.2).

We obtain then the analogue for $M_{\Lambda}^{2}$ of [6, Corollary 2.7]. 
Corollary 3.2. Suppose $\rho: \mathbb{R}_{+} \rightarrow \mathbb{R}_{+}$is an increasing $C^{1}$ function with $\rho(0)=0$, such that $\int_{0}^{1}(\psi(x))^{2} \rho^{\prime}(1-x) d x<\infty$. If $\mu\left(J_{\epsilon}\right) \leq \rho(\epsilon)$ for all $\epsilon \in(0,1]$, then $\mu$ is $\Lambda$-embedding.

Proof. Similar to the $L^{1}$ case, the proof follows from Theorem 3.1 by applying Lemma 2.7 to $g=\psi^{2}$.

More interesting, we may improve Proposition 2.6 (ii): if the support of $\mu$ is compact in $[0,1)$, then the embedding is not only compact, but inside any Schattenvon Neumann class.

Theorem 3.3. If $\operatorname{supp} \mu \subset[0,1-\varepsilon]$, then $i_{\mu} \in \mathcal{S}_{q}$ for any $q>0$.

Proof. Denote $b=1-\epsilon$. We have $\int \psi(x)^{2} d \mu(x) \leq \psi(b)^{2}\|\mu\|$, and thus, by Theorem 3.1

$$
\left\|i_{\mu}\right\| \leq \psi(b) \sqrt{\|\mu\|} .
$$

Let us fix a positive integer $k$ and a number $b<b^{\prime}<1$. If $f \in M_{\Lambda}^{2}$, then, by (3.2), we have

$$
\int_{0}^{b^{\prime}}\left|f^{(k)}(x)\right|^{2} d x \leq\left(\psi^{(k)}\left(b^{\prime}\right)\right)^{2}\|f\|^{2},
$$

and thus the $k$ times differentiation operator $D_{k}$ is bounded from $M_{\Lambda}^{2}$ to $L^{2}\left(\left[0, b^{\prime}\right]\right)$, of norm at most $\psi^{(k)}\left(b^{\prime}\right)$.

On the other hand, integration is a Hilbert-Schmidt operator on $L^{2}\left(\left[0, b^{\prime}\right]\right)$, of $\mathcal{S}_{2}$ norm $b^{\prime} / \sqrt{2} \leq 2^{-1 / 2}$. It follows by Lemma 2.2 (i) that $k$ times integration is an operator $J_{k}$ in $\mathcal{S}_{2 / k}$ on $L^{2}\left(\left[0, b^{\prime}\right]\right)$, of norm at most $2^{-k / 2}$. The composition $R_{k}=J_{k} \circ D_{k}$ is, modulo a finite rank operator, the restriction of functions in $M_{\Lambda}^{2}$ to $\left[0, b^{\prime}\right]$; it is thus (again by Lemma $2.2(\mathrm{i})$ ) an operator of class $\mathcal{S}_{2 / k}$ from $M_{\Lambda}^{2}$ to $L^{2}\left(\left[0, b^{\prime}\right]\right)$, whose image is in $M_{\Lambda, b^{\prime}}^{2}$, and

$$
\left\|R_{k}\right\|_{2 / k} \leq 2^{-k / 2} \psi^{(k)}\left(b^{\prime}\right) \text {. }
$$

Consider then the embedding $i_{\mu}^{\prime}$ from $M_{\Lambda, b^{\prime}}^{2}$ into $L^{2}(\mu)$. According to (3.3) and (3.4), $i_{\mu}^{\prime}$ is bounded and

$$
\left\|i_{\mu}^{\prime}\right\| \leq \psi_{b^{\prime}}(b) \sqrt{\|\mu\|}=b^{\prime-1 / 2} \psi\left(b / b^{\prime}\right) \sqrt{\|\mu\|} .
$$

Finally, $i_{\mu}=i_{\mu}^{\prime} R_{k}$, and thus

$$
\left\|i_{\mu}\right\|_{2 / k} \leq 2^{-k / 2} b^{\prime-1 / 2} \psi^{(k)}\left(b^{\prime}\right) \psi\left(b / b^{\prime}\right) \sqrt{\|\mu\|} .
$$

Choosing $k$ such that $2 / k \leq q$, inequality (3.5) proves the theorem for any $q>0$.

If $\mu$ is a general measure, Theorem 3.3 can still be used in order to obtain sufficient conditions for the embedding to be in $\mathcal{S}_{2}$. Namely, we take a sequence $b_{n} \nearrow 1$ and define $\mu_{j}=\mu \mid\left[b_{j}, b_{j+1}\right)$; then $i_{\mu}^{2}=\sum_{j} i_{\mu_{j}}^{2}$, and thus we have (for $q \geq 1$ ) $\left\|i_{\mu}^{2}\right\|_{q} \leq\left\|\sum_{j} i_{\mu_{j}}^{2}\right\|_{q}$. We may then apply Theorem 3.3 to each of the measures $\mu_{j}$. The statements obtained depend on the arbitrary sequence $\left(b_{j}\right)$, and are thus not very natural. We prefer to state a more elegant result, valid for Hilbert-Schmidt embeddings.

Theorem 3.4. Define $\Psi(x)=\psi^{\prime}\left(x^{1 / 4}\right) \psi\left(x^{1 / 4}\right)$. If $\Psi \in L^{2}(\mu)$, then $i_{\mu} \in \mathcal{S}_{2}$. 
Proof. Consider the sequence $b_{n}$ defined by $b_{0}=0, b_{1}=1 / 2$, and $b_{j+1}=\sqrt{b_{j}}$; obviously it is an increasing sequence tending to 1 . Define also, for $j \geq 0, \mu_{j}=$ $\mu \mid\left[b_{j}, b_{j+1}\right)$; we have

$$
L^{2}(\mu)=\bigoplus_{j=0}^{\infty} L^{2}\left(\mu_{j}\right)
$$

We have $\left\|i_{\mu}\right\|_{2}^{2}=\sum_{j=0}^{\infty}\left\|i_{\mu_{j}}\right\|_{2}^{2}$. By Theorem 3.3 applied with $b=b_{j+1}$ and $b^{\prime}=$ $b_{j+2}$, we have, for some constant $C>0$,

$$
\begin{aligned}
\left\|i_{\mu_{j}}\right\|_{2}^{2} & \leq C\left(\psi^{\prime}\left(b_{j+2}\right)\right)^{2}\left(\psi\left(\frac{b_{j+1}}{b_{j+2}}\right)\right)^{2}\left\|\mu_{j}\right\|=C\left(\psi^{\prime}\left(b_{j+2}\right)\right)^{2}\left(\psi\left(b_{j+2}\right)\right)^{2}\left\|\mu_{j}\right\| \\
& =C\left(\Psi\left(b_{j}\right)\right)^{2}\left\|\mu_{j}\right\| .
\end{aligned}
$$

Since $\Psi$ is increasing, the last term is less than or equal to $C \int \Psi(x)^{2} d \mu_{j}(x)$. Therefore

$$
\left\|i_{\mu}\right\|_{2}^{2}=\sum_{j=0}^{\infty}\left\|i_{\mu_{j}}\right\|_{2}^{2} \leq C \sum_{j=0}^{\infty} \int \Psi(x)^{2} d \mu_{j}(x)=\int \Psi(x)^{2} d \mu(x),
$$

which proves the theorem.

The function $\psi$ is hard to compute precisely, but one can give estimates that can be used in the above embedding results. Here are some examples; the estimates for the power series are classical and can be obtained, for instance, by the techniques in [5].

(1) Suppose $\lambda_{n}=2^{n}$ (a typical case of a lacunary sequence). Then $\left(2^{n / 2} x^{2^{n}}\right)$ forms a Riesz basis of $M_{\Lambda}^{2}$, so in (3.1) we have $d_{n} \sim 2^{-n / 2}$, and

$$
\psi(x) \sim \sum_{n=1}^{\infty} 2^{n / 2} x^{2^{n}} \sim \frac{1}{\sqrt{1-x}} .
$$

This estimate shows the fact that the function $\psi$ reflects only partially the properties of the sequence $\Lambda$. It does not use the precise fact that $\left(2^{n / 2} x^{2^{n}}\right)$ is a Riesz basis, but only that it is a uniformly minimal sequence. For instance, one cannot use it to recapture the results obtained in Section 4 below.

(2) $\lambda_{n}=n^{2}$. This is a typical case of what is called a "standard" sequence (which is defined by the fact that $\lambda_{n+1} / \lambda_{n} \rightarrow 1$ ). A computation essentially done in [6, Section 7] shows that

$$
\psi(x) \sim e^{\frac{C}{1-x}}
$$

for some constant $C>0$.

\section{Sublinear MEASURES}

As in [6], one can obtain much more precise results if one considers special classes of measures. In this section we will again consider different values of $p$, so we return to the notation $i_{\mu}^{p}$ and $M_{\Lambda}^{p}$.

Definition 4.1. A measure $\mu$ is called sublinear if there is a constant $C>0$ such that for any $0<\varepsilon<1$ we have $\mu\left(J_{\varepsilon}\right) \leq C \varepsilon$. The smallest such $C$ will be denoted by $\|\mu\|_{S}$. The measure $\mu$ is called vanishing sublinear if $\lim _{\varepsilon \rightarrow 0} \frac{\mu\left(J_{\varepsilon}\right)}{\varepsilon}=0$. 
As one can see, sublinear measures satisfy the condition of Corollary 3.2 for $\rho(\epsilon)=\epsilon$. The next lemma gathers some results that are either contained in or analogues of [6, Section 4].

Lemma 4.2. (i) Suppose that $\|\mu\|_{S}<\infty$. If $g$ is continuous, positive, and increasing, we have

$$
\int_{[0,1]} g d \mu \leq\|\mu\|_{S} \int_{[0,1]} g d m .
$$

(ii) Suppose $1 \leq p<\infty$. If there exists $M>0$ such that $\frac{\lambda_{n+1}}{\lambda_{n}} \leq M$, then any $\Lambda_{p}$-embedding measure is sublinear.

(iii) If $\mu$ is sublinear, $1 \leq p<\infty$, then

$$
\sup _{\lambda}\left\|(p \lambda+1)^{1 / p} x^{\lambda}\right\|_{L^{p}(\mu)} \leq\|\mu\|_{S}^{1 / p}
$$

Precise results can be obtained for sublinear measures if we also assume that the sequence $\Lambda$ is lacunary.

Theorem 4.3. Suppose $\Lambda$ is lacunary. If $\mu$ is a sublinear measure, then $\mu$ is $\Lambda_{2}$-embedding and $\left\|i_{\mu}^{2}\right\|$ is of order $\|\mu\|_{S}^{1 / 2}$.

Proof. Since $\Lambda$ is lacunary, the sequence of functions $g_{n}=\lambda_{n}^{1 / 2} x^{\lambda_{n}}$ forms a Riesz basis in $M_{\Lambda}^{2}$. The embedding $i_{\mu}^{2}: M_{\Lambda}^{2} \rightarrow L^{2}(\mu)$ is defined by $i_{\mu}^{2}\left(g_{n}\right)=g_{n}$.

We have

$$
\left\langle g_{n}, g_{m}\right\rangle_{L^{2}(\mu)}=\lambda_{n}^{1 / 2} \lambda_{m}^{1 / 2} \int_{[0,1]} x^{\lambda_{n}+\lambda_{m}} d \mu .
$$

Since $\mu$ is sublinear and the function $x^{\lambda_{n}+\lambda_{m}}$ is continuous, positive, and increasing, it follows from Lemma 2.7 that

$$
\int_{[0,1]} x^{\lambda_{n}+\lambda_{m}} d \mu \leq\|\mu\|_{S} \int_{[0,1]} x^{\lambda_{n}+\lambda_{m}} d m,
$$

and thus

$$
\left\langle g_{n}, g_{m}\right\rangle_{L^{2}(\mu)} \leq\|\mu\|_{S}\left\langle g_{n}, g_{m}\right\rangle_{2} .
$$

Thus, if we define the matrices $A=\left(\left\langle g_{n}, g_{m}\right\rangle_{L^{2}(\mu)}\right)$ and $B=\left(\|\mu\|_{S}\left\langle g_{n}, g_{m}\right\rangle_{2}\right)$, then the entries of $A$ are nonnegative and majorized by those of $B$. But $B$ is bounded since $\left(g_{n}\right)$ is a Riesz basis in $M_{\Lambda}^{2}$ (by Lemma 2.1 (i)); it follows that $A$ is also bounded. Therefore, by 2.1 (ii), the embedding $i_{\mu}^{2}$ is bounded, of norm of order $\|\mu\|_{S}^{1 / 2}$.

Combining Lemma 4.2 (ii) with Theorem 4.3, we obtain

Corollary 4.4. If $\Lambda$ is lacunary with $\frac{\lambda_{n+1}}{\lambda_{n}} \leq M$ for some $M>0$, then a measure $\mu$ is $\Lambda_{2}$-embedding if and only if it is sublinear.

Using Theorem 4.3 we get vanishing sublinearity as a sufficient condition for compactness of the embedding.

Corollary 4.5. If $\Lambda$ is lacunary, then for any vanishing sublinear measure $\mu$ the embedding $i_{\mu}: M_{\Lambda}^{2} \rightarrow L^{2}(\mu)$ is compact. 
Proof. Recalling that $\mu_{m}$ is the measure equal to $\mu$ on $\left[0, \frac{1}{m}\right]$ and equal to 0 elsewhere on $[0,1]$, we can view $i_{\mu_{m}}$ as the embeddings $M_{\Lambda}^{2} \hookrightarrow L^{2}\left(\mu_{m}\right)$ and regard $L^{2}\left(\mu_{m}\right)$ as a subspace of $L^{2}(\mu)$. Then $\mu_{m}^{\prime}=\mu-\mu_{m}$ is the measure $\mu$ restricted to $J_{\frac{1}{m}}$. By vanishing sublinearity and Theorem 4.3, $\mu_{m}^{\prime}$ is $\Lambda$-embedding with embedding constants $\left\|\mu_{m}^{\prime}\right\|_{S} \rightarrow 0$ as $m \rightarrow \infty$. Therefore

$$
\left\|i_{\mu}-i_{\mu_{m}}\right\|=\left\|i_{\mu_{m}^{\prime}}\right\|=\left\|\mu_{m}^{\prime}\right\|_{S} \longrightarrow 0
$$

as $m \rightarrow \infty$. Since $i_{\mu_{m}}$ is compact by Proposition 2.6 (ii), it follows that $i_{\mu}$ is compact.

\section{INTERPOLATION}

If $\Lambda$ is lacunary and $\mu$ is sublinear, then $\mu$ is $\Lambda_{2}$-embedding by Theorem 4.3 , while it is $\Lambda_{1}$-embedding by Theorem 5.5 from [6]. It is interesting that although the Müntz spaces do not form an interpolation scale of spaces, we may still apply the proof of the Riesz-Thorin theorem in order to extend the result to values $1<p<2$. We will actually obtain below a more general result concerning interpolation of embeddings.

Theorem 5.1. Suppose $1 \leq p_{0}<p_{1}<\infty$. For $0<t<1$, define $p_{t}$ by

$$
\frac{1}{p_{t}}=\frac{1-t}{p_{0}}+\frac{t}{p_{1}}
$$

If a positive measure $\mu$ on $[0,1]$ is $\Lambda_{p_{0}}$-embedding and $\Lambda_{p_{1}}$-embedding, then it is also $\Lambda_{p_{t}}$-embedding with

$$
\left\|i_{\mu}\right\|_{p_{t}} \leq\left\|i_{\mu}\right\|_{p_{0}}^{1-t}\left\|i_{\mu}\right\|_{p_{1}}^{t}
$$

where $\left\|i_{\mu}\right\|_{p_{s}}$ is the operator norm of $i_{\mu}: M_{\Lambda}^{p_{s}} \rightarrow L^{p_{s}}(\mu)$ for $0 \leq s \leq 1$.

Proof. The proof follows the Riesz-Thorin Theorem (see, for instance, [3]), so we will just sketch the main steps. For any $1 \leq p \leq \infty$, denote, as customary, by $p^{\prime}$ the conjugate exponent (satisfying $1 / p+1 / p^{\prime}=1$ ). Let $P_{\Lambda}$ be the space of all polynomials in $\operatorname{span}\left\{x^{\lambda}: \lambda \in \Lambda\right\}$. Then $P_{\Lambda}$ is dense in $M_{\Lambda}^{p}$ and the theorem will be proved once we show that

for all $f \in P_{\Lambda}$.

$$
\|f\|_{L^{p_{t}(\mu)}} \leq\left\|i_{\mu}\right\|_{p_{0}}^{1-t}\left\|i_{\mu}\right\|_{p_{1}}^{t}\|f\|_{p_{t}}
$$

Fix then $f \in P_{\Lambda}$ with $\|f\|_{p_{t}}=1$. If $p_{t}^{\prime}$ is the exponent conjugate to $p_{t}$, then

$$
\|f\|_{L^{p_{t}(\mu)}}=\sup \left\{\left|\int_{[0,1]} f g d \mu\right|: g \text { continuous, }\|g\|_{L^{p_{t}^{\prime}(\mu)}}=1\right\} \text {. }
$$

Take then $g$ continuous with $\|g\|_{L^{p_{t}^{\prime}(\mu)}}=1$. Define, for $0 \leq \Re z \leq 1$,

$$
\frac{1}{p(z)}=\frac{1-z}{p_{0}}+\frac{z}{p_{1}}
$$

and, for $0 \leq x \leq 1, \phi(z, x)=|f(x)|^{p_{t} / p(z)-1} f(x), \psi(z, x)=|g(x)|^{p_{t}^{\prime} / p^{\prime}(z)-1} g(x)$, $F(z)=\int \phi(z, x) \psi(z, x) d \mu$. Then $F(z)$ is analytic on $0<\Re z<1$, bounded and continuous on $0 \leq \Re z \leq 1$. From $\|f\|_{p_{t}}=1$ and $\|g\|_{L^{p_{t}^{\prime}(\mu)}}=1$ it follows that

$$
\|\phi(i s)\|_{p_{0}}=\|\phi(1+i s)\|_{p_{1}}=\|\psi(i s)\|_{L^{p_{0}^{\prime}(\mu)}}=\|\psi(1+i s)\|_{L^{p_{1}^{\prime}(\mu)}}=1,
$$

which implies, by Hölder's inequality,

$$
|F(i s)| \leq\|\phi(i s)\|_{L^{p_{0}(\mu)}}\|\psi(i s)\|_{L^{p_{0}^{\prime}(\mu)}} \leq\left\|i_{\mu}^{p_{0}}\right\|\|\phi(i s)\|_{p_{0}}\|\psi(i s)\|_{L^{p_{0}^{\prime}(\mu)}}=\left\|i_{\mu}^{p_{0}}\right\|,
$$


and similarly $|F(1+i s)| \leq\left\|i_{\mu}^{p_{1}}\right\|$. The Three Lines Lemma then yields

$$
\left|\int f(x) g(x) d \mu(x)\right|=|F(t)| \leq\left\|i_{\mu}^{p_{0}}\right\|^{1-t}\left\|i_{\mu}^{p_{1}}\right\|^{t},
$$

whence the theorem follows by (5.1).

Corollary 5.2. If $\Lambda$ is lacunary, then:

(i) every sublinear measure $\mu$ is $\Lambda_{p}$-embedding for $1 \leq p \leq 2$;

(ii) for any vanishing sublinear measure $\mu$ the embedding $i_{\mu}^{p}: M_{\Lambda}^{p} \rightarrow L^{p}(\mu)$ is compact for $1 \leq p \leq 2$.

\section{Schatten-von Neumann embeddings}

We return now to the case $p=2$ and the simplified notation of Section 3 , A slight strengthening of the sublinearity condition implies that for quasilacunary sequences the embedding belongs already to all Schatten-von Neumann classes.

Theorem 6.1. If $\Lambda$ is quasilacunary and the positive measure $\mu$ satisfies $\mu\left(J_{\varepsilon}\right) \leq$ $C \varepsilon^{\alpha}$ for some $\alpha>1$, then $i_{\mu} \in \mathcal{S}_{q}$ for all $q>0$.

Proof. Again the letter $C$ will be used for possibly different universal constants. Suppose then that $\left\{n_{k}\right\}$ is a sequence of integers with $N:=\sup _{k}\left(n_{k+1}-n_{k}\right)<\infty$, such that for some $\gamma>1$ we have $\gamma \leq \lambda_{n_{k+1}} / \lambda_{n_{k}+1} \leq \gamma^{2(N-1)}$. With the notation of Lemma 2.8, each subspace $F_{k}$ has dimension $n_{k+1}-n_{k}$. If we choose an orthonormal basis in each of the spaces $F_{k}$, it follows easily from Lemma 2.8 that the union of these bases is a Riesz basis in $M_{\Lambda}^{2}$. Let us denote by $\left(\phi_{i}\right)$ this basis; we may also assume it consists of real functions.

Suppose $\phi_{i} \in F_{k}$. Applying Lemma 2.4 to $\phi_{i}$ with $\beta_{j}=\beta=1 /\left(n_{k+1}-n_{k}\right)$ and Lemma 2.7 for $g(x)=x^{2 \beta \lambda_{n_{k}+1}}$ and $\rho(x)=C x^{\alpha}$, we obtain

$$
\begin{aligned}
& \left\|i_{\mu} \phi_{i}\right\|_{L^{2}(\mu)}^{2}=\int_{0}^{1}\left|\phi_{i}(x)\right|^{2} d \mu(x) \leq 4\left\|\phi_{i}\right\|_{\infty}^{2} \int_{0}^{1}\left(\sum_{j=n_{k}+1}^{n_{k+1}} x^{\lambda_{j} \beta_{j}}\right)^{2} d \mu(x) \\
& \quad \leq 4^{(N+1)}\left\|\phi_{i}\right\|_{\infty}^{2} \sum_{j=n_{k}+1}^{n_{k+1}} \int_{0}^{1} x^{2 \lambda_{j} \beta_{j}} d \mu(x) \leq 4^{(N+1)}\left\|\phi_{i}\right\|_{\infty}^{2} N \int_{0}^{1} x^{2 \beta \lambda_{n_{k}+1}} d \mu(x) \\
& \quad \leq 4^{(N+1)}\left\|\phi_{i}\right\|_{\infty}^{2} N \int_{0}^{1} x^{2 \beta \lambda_{n_{k}+1}}\left(\alpha C(1-x)^{\alpha-1}\right) d x \\
& \quad \leq C\left\|\phi_{i}\right\|_{\infty}^{2} B\left(2 \beta \lambda_{n_{k}+1}+1, \alpha\right) .
\end{aligned}
$$

The Euler beta function satisfies the following asymptotic formula: if $t$ is large and $s$ is fixed, then

Therefore

$$
B(t, s)=\int_{0}^{1} x^{t-1}(1-x)^{s-1} d x \sim \Gamma(s) t^{-s} .
$$

$$
\left\|i_{\mu} \phi_{i}\right\|_{L^{2}(\mu)}^{2} \leq C\left\|\phi_{i}\right\|_{\infty}^{2} \Gamma(\alpha)\left(2 \beta \lambda_{n_{k}+1}+1\right)^{-\alpha} \leq C \frac{\left\|\phi_{i}\right\|_{\infty}^{2}}{\lambda_{n_{k}+1}^{\alpha}} .
$$

It is elementary to see (and can be found in [6], Lemma 5.4) that if $f:[0,1] \rightarrow \mathbb{R}$ is a nonconstant differentiable function, then

$$
\|f\|_{1} \geq \min \left\{\frac{\|f\|_{\infty}}{4}, \frac{\|f\|_{\infty}^{2}}{2\left\|f^{\prime}\right\|_{\infty}}\right\}
$$


We apply this inequality to $f=\phi_{i}^{2}$. If the minimum is given by the first term above, then $\left\|\phi_{i}\right\|_{\infty}^{2} \leq 4\left\|\phi_{i}\right\|_{2}^{2}=4$, whence from (6.1) it follows that

$$
\left\|i_{\mu} \phi_{i}\right\|_{L^{2}(\mu)} \lesssim \frac{4}{\lambda_{n_{k}}^{\alpha / 2}} \lesssim \frac{1}{\gamma^{\alpha(k-1) / 2}}
$$

If the minimum is given by the second term, that is, $\|f\|_{\infty}^{2} \leq 2\|f\|_{1}\left\|f^{\prime}\right\|_{\infty}$, noting that $\|f\|_{\infty}=\left\|\phi_{i}\right\|_{\infty}^{2},\|g\|_{1}=\left\|\phi_{i}\right\|_{2}^{2}=1$, and $f^{\prime}=2 \phi_{i} \phi_{i}^{\prime}$, we obtain, using Lemma 2.5.

$$
\left\|\phi_{i}\right\|_{\infty}^{4} \leq 4\left\|\phi_{i}\right\|_{2}^{2}\left\|\phi_{i}^{\prime}\right\|_{\infty}\left\|\phi_{i}\right\|_{\infty} \leq 4 K N \lambda_{n_{k+1}}\left\|\phi_{i}\right\|_{\infty}^{2}
$$

Therefore $\left\|\phi_{i}\right\|_{\infty} \leq C \lambda_{n_{k+1}}^{1 / 2}$, whence, again by (6.1),

$$
\left\|i_{\mu} \phi_{i}\right\|_{L^{2}(\mu)} \leq C \frac{\left\|\phi_{i}\right\|_{\infty}}{\lambda_{n_{k}+1}^{\alpha / 2}} \leq C \frac{\lambda_{n_{k+1}}^{1 / 2}}{\lambda_{n_{k}+1}^{\alpha / 2}} \leq C\left(\frac{\lambda_{n_{k+1}}}{\lambda_{n_{k}+1}}\right)^{1 / 2} \frac{1}{\lambda_{n_{k}+1}(\alpha-1) / 2} .
$$

The factor $\left(\lambda_{n_{k+1}} / \lambda_{n_{k}+1}\right)^{1 / 2}$ is bounded by $\gamma^{N-1}$, and therefore

$$
\left\|i_{\mu} \phi_{i}\right\|_{L^{2}(\mu)} \leq C \frac{1}{\lambda_{n_{k}^{2}}^{\frac{\alpha-1}{2}}} \leq C \frac{1}{\gamma^{\frac{(\alpha-1)(k-1)}{2}}} .
$$

Note that the inequalities (6.2) and (6.3) have been obtained for $i=n_{k}+$ $1, \ldots, n_{k+1}$. They imply, if $q \leq 2$, that

$$
\begin{aligned}
\sum_{i=1}^{\infty}\left\|i_{\mu} \phi_{i}\right\|_{L^{2}(\mu)}^{q} & =\sum_{k=0}^{\infty} \sum_{i=n_{k}+1}^{n_{k+1}}\left\|i_{\mu} \phi_{i}\right\|_{L^{2}(\mu)}^{q} \\
& \leq C N\left(\sum_{k=0}^{\infty} \frac{1}{\left(\gamma^{\alpha q / 2}\right)^{k-1}}+\sum_{k=0}^{\infty} \frac{1}{\left(\gamma^{(\alpha-1) q / 2}\right)^{k-1}}\right)<\infty .
\end{aligned}
$$

By Corollary 2.3 (ii), it follows that $i_{\mu} \in \mathcal{S}_{q}\left(M_{\Lambda}^{2}, L^{2}(\mu)\right)$ for all $q \leq 2$, and therefore for all $q>0$.

In particular, for $\Lambda$ lacunary the condition $\mu\left(J_{\varepsilon}\right) \leq C \varepsilon^{\alpha}$ for some $\alpha>1$ implies that the embedding is in all Schatten-von Neumann classes.

\section{EXAMPLES}

7.1. In the first example we intend to construct a measure $\mu$ and a sequence $\Lambda$ such that $\mu$ is $\Lambda_{p}$-embedding for $p=2$ but not for $p=1$. As above, we will use the same letter $C$ for possibly different universal constants.

Take $\mu=\sum_{k} c_{k} \delta_{a_{k}}$, with $0<a_{k}<1$. We will define recurrently $\lambda_{n} \rightarrow \infty$, $a_{n} \rightarrow 1$, and $c_{n} \rightarrow 0$ such as to have:

(A) $\sup _{n} \lambda_{n} c_{n} a_{n}^{\lambda_{n}}=\infty$;

(B) $\sum_{k} \lambda_{n} c_{k} a_{k}^{2 \lambda_{n}} \leq C \frac{\ln n}{n^{2}}$.

First, one can start with $\lambda_{1}=1, a_{1}=1 / 2$ and $c_{1}=1$. Suppose then that $\lambda_{k}, a_{k}, c_{k}$ have been obtained for $k \leq n-1$. Choose first $\lambda_{n}$ sufficiently large such that

(i) $\lambda_{n} \sum_{k \leq n-1} a_{k}^{\lambda_{n}} \leq \frac{1}{n^{2}}$;

(ii) $\lambda_{n+1} \geq n^{4} \lambda_{n}$. 
Put $a_{n}=1-\frac{2 \ln n}{\lambda_{n}}$ and $c_{n}=\frac{2 n^{2} \ln n}{\lambda_{n}}$. Then

$$
a_{n}^{\lambda_{n}}=\left(1-\frac{2 \ln n}{\lambda_{n}}\right)^{\lambda_{n}} \sim e^{-2 \ln n}=\frac{1}{n^{2}},
$$

whence $\lambda_{n} c_{n} a_{n}^{\lambda_{n}} \sim \ln n$ and thus (A) is satisfied.

To achieve (B), write

$$
\sum_{k} \lambda_{n} c_{k} a_{k}^{2 \lambda_{n}}=\sum_{k \leq n-1} \lambda_{n} c_{k} a_{k}^{2 \lambda_{n}}+\lambda_{n} c_{n} a_{n}^{2 \lambda_{n}}+\sum_{k \geq n+1} \lambda_{n} c_{k} a_{k}^{2 \lambda_{n}} .
$$

The first sum is smaller than $\frac{1}{n^{2}}$ by (i). The second term is of order $\frac{\ln n}{n^{2}}$ by (7.1) and the definition of $c_{n}$. For the third term, we have

$$
\sum_{k \geq n+1} \lambda_{n} c_{k} a_{k}^{2 \lambda_{n}} \leq \lambda_{n} \sum_{k \geq n+1} c_{k} .
$$

From (ii) it follows in particular that $c_{n}$ decreases faster than a geometric progression (which also proves the convergence of the sum defining $\mu$ ), and thus for some constant $C$ we have

$$
\sum_{k \geq n+1} c_{k} \leq C c_{n+1}=\frac{C 2(n+1)^{2} \ln (n+1)}{\lambda_{n+1}} .
$$

Applying again (ii),

$$
\lambda_{n} \sum_{k \geq n+1} c_{k} \leq C \frac{\ln n}{n^{2}} .
$$

So we have estimated all three terms of (17.2) by $\frac{\ln n}{n^{2}}$, whence (B) is satisfied.

Now (ii) implies that $\Lambda$ is lacunary and the functions $g_{k}(x)=\lambda_{k}^{1 / 2} x^{\lambda_{k}}$ form a Riesz basis in $M_{\Lambda}^{2}$. Thus

$$
\left\|\sum_{k} b_{k} g_{k}\right\|_{2}^{2} \sim \sum_{k}\left|b_{k}\right|^{2}
$$

On the other hand,

$$
\left\|\sum_{k} b_{k} g_{k}\right\|_{L^{2}(\mu)}^{2} \leq\left(\sum_{k}\left|b_{k}\right|\left\|g_{k}\right\|_{L^{2}(\mu)}\right)^{2} \leq\left(\sum_{k}\left|b_{k}\right|^{2}\right)\left(\sum_{k}\left\|g_{k}\right\|_{L^{2}(\mu)}^{2}\right) .
$$

According to (B), we have

$$
\left\|g_{n}\right\|_{L^{2}(\mu)}^{2}=\sum_{k} c_{k} \lambda_{n} a_{k}^{2 \lambda_{n}} \leq C \frac{\ln n}{n^{2}},
$$

and thus $\sum_{k}\left\|g_{k}\right\|_{L^{2}(\mu)}^{2}<\infty$. So it follows from (7.3) and (17.4) that

$$
\left\|\sum_{k} b_{k} g_{k}\right\|_{L^{2}(\mu)}^{2} \leq C\left(\sum_{k}\left\|g_{k}\right\|_{L^{2}(\mu)}^{2}\right)\left(\left\|\sum_{k} b_{k} g_{k}\right\|_{2}^{2}\right) .
$$

Thus $\mu$ is $\Lambda_{2}$-embedding.

On the other side, for $p=1$,

$$
\left\|\lambda_{n} x^{\lambda_{n}}\right\|_{L^{1}(\mu)}=\int_{[0,1]} \lambda_{n} x^{\lambda_{n}} d \mu=\sum_{k} c_{k} \lambda_{n} a_{k}^{\lambda_{n}} \geq c_{n} \lambda_{n} a_{n}^{\lambda_{n}} .
$$


So by $(\mathrm{A})$,

$$
\sup _{n}\left\|\lambda_{n} x^{\lambda_{n}}\right\|_{L^{1}(\mu)} \geq \sup _{n} c_{n} \lambda_{n} a_{n}^{\lambda_{n}}=\infty
$$

whence $\left\|\lambda_{n} x^{\lambda_{n}}\right\|_{1} \leq 1$ for all $n=1,2, \ldots$ Hence $\mu$ is not $\Lambda_{1}$-embedding.

7.2. In this example we consider the Hilbert space $M_{\Lambda}^{2}$. We will show that for any $0<r<q$ we can construct a lacunary sequence $\Lambda$ and a measure $\mu$ such that $i_{\mu}^{2} \notin \mathcal{S}_{r}$ but $i_{\mu}^{2} \in \mathcal{S}_{q}$.

Fix $q>r>0$. Choose a sequence $\left\{\alpha_{n}\right\} \in \ell^{q}$ with $\left|\alpha_{n}\right|<1$ but $\left\{\alpha_{n}\right\} \notin \ell^{r}$, and a double sequence $\left\{\beta_{n m}\right\}_{n, m=1}^{\infty}$ with $\sum_{n} \sum_{m} \beta_{n m}<\frac{1}{4}$.

The measure will again be of the form $\mu=\sum_{j} c_{j} \delta_{a_{j}}$, with $0<a_{j}<1$; we will construct $a_{n}, c_{n}$ as well as $\lambda_{n}$ recurrently. Suppose $a_{j}, c_{j}, \lambda_{j}$ have been obtained for $j \leq n-1$. Choose first $\lambda_{n}$ sufficiently large such that $\Lambda$ is lacunary, and

$$
\begin{aligned}
\sum_{i=1}^{n-1} c_{i} \lambda_{n} a_{i}^{2 \lambda_{n}} & \leq \frac{1}{8} \alpha_{n}^{2}, \\
\sum_{i=1}^{n-1} c_{i} \lambda_{j}^{1 / 2} \lambda_{n}^{1 / 2} a_{i}^{\lambda_{j}+\lambda_{n}} & \leq \frac{1}{4} \alpha_{j} \alpha_{n} \beta_{j n}^{1 / 2} \quad \text { for } j=1, \ldots, n-1, \\
\frac{\alpha_{n}^{2} \lambda_{i}^{1 / 2} \lambda_{j}^{1 / 2}}{\lambda_{n}} & \leq \frac{1}{2^{n+2-\max \{i, j\}}} \alpha_{i} \alpha_{j} \beta_{i j}^{1 / 2} \quad \text { for } i, j<n, \\
\frac{\alpha_{n}^{2} \lambda_{i}^{1 / 2}}{\lambda_{n}^{1 / 2}} & \leq \frac{1}{2} \alpha_{i} \alpha_{n} \beta_{i n}^{1 / 2} \quad \text { for } i, j<n .
\end{aligned}
$$

Then take $a_{n}=e^{-1 / 2 \lambda_{n}}$ and $c_{n}=\frac{\alpha_{n}^{2}}{\lambda_{n}}$; we have $c_{n} \lambda_{n} a_{n}^{2 \lambda_{n}}=\frac{\alpha_{n}^{2}}{e}$.

Now consider the Riesz basis $\left(g_{n}\right)=\left(\lambda_{n}^{1 / 2} x^{\lambda_{n}}\right)$ for $M_{\Lambda}^{2}$. By (7.5) and (7.7), we have

$$
\begin{aligned}
\left\|i_{\mu}^{2} g_{n}\right\|_{L^{2}(\mu)}^{2} & =\int_{[0,1]} \lambda_{n} x^{2 \lambda_{n}} d \mu=\sum_{j} c_{j} \lambda_{n} a_{j}^{2 \lambda_{n}} \\
& =\sum_{j \leq n-1} c_{j} \lambda_{n} a_{j}^{2 \lambda_{n}}+c_{n} \lambda_{n} a_{n}^{2 \lambda_{n}}+\sum_{j \geq n+1} c_{j} \lambda_{n} a_{j}^{2 \lambda_{n}} \\
& \leq \frac{1}{8} \alpha_{n}^{2}+\alpha_{n}^{2}+\sum_{j \geq n+1} \frac{\alpha_{j}^{2} \lambda_{n} a_{j}^{2 \lambda_{n}}}{\lambda_{j}} \leq \frac{1}{8} \alpha_{n}^{2}+\alpha_{n}^{2}+\sum_{j \geq n+1} \frac{1}{2^{j+2-n}} \alpha_{n}^{2} \beta_{n n}^{1 / 2} \\
& \leq \frac{1}{8} \alpha_{n}^{2}+\alpha_{n}^{2}+\frac{1}{4} \alpha_{n}^{2}<\frac{3}{2} \alpha_{n}^{2} .
\end{aligned}
$$

Since clearly $\left\|i_{\mu}^{2} g_{n}\right\|_{L^{2}(\mu)}^{2} \geq c_{n} \lambda_{n} a_{n}^{2 \lambda_{n}}$ we obtain

$$
\frac{1}{e} \alpha_{n}^{2} \leq\left\|i_{\mu}^{2} g_{n}\right\|_{L^{2}(\mu)}^{2} \leq \frac{3}{2} \alpha_{n}^{2}
$$

If we define $f_{n}=i_{\mu}^{2} g_{n} /\left\|i_{\mu}^{2} g_{n}\right\|_{L^{2}(\mu)}$, then the $f_{n}$ are nonnegative functions, and (7.9) implies that $\left\langle f_{n}, f_{m}\right\rangle_{L^{2}(\mu)} \leq e \alpha_{n}^{-1} \alpha_{m}^{-1} \lambda_{n}^{1 / 2} \lambda_{m}^{1 / 2} \int_{[0,1]} x^{\lambda_{n}+\lambda_{m}} d \mu(x)$. The 
last quantity can be estimated using (7.6), (7.7), and (7.8); we have, for $n>m$,

$$
\begin{aligned}
& e \alpha_{n}^{-1} \alpha_{m}^{-1} \lambda_{n}^{1 / 2} \lambda_{m}^{1 / 2} \int_{[0,1]} x^{\lambda_{n}+\lambda_{m}} d \mu(x)=e \alpha_{n}^{-1} \alpha_{m}^{-1} \lambda_{n}^{1 / 2} \lambda_{m}^{1 / 2} \sum_{j} c_{j} a_{j}^{\lambda_{n}+\lambda_{m}} \\
& =e \alpha_{n}^{-1} \alpha_{m}^{-1}\left(\sum_{j \leq n-1} c_{j} \lambda_{n}^{1 / 2} \lambda_{m}^{1 / 2} a_{j}^{\lambda_{n}+\lambda_{m}}+c_{n} \lambda_{n}^{1 / 2} \lambda_{m}^{1 / 2} a_{n}^{\lambda_{n}+\lambda_{m}}\right. \\
& \left.\quad+\sum_{j \geq n+1} c_{j} \lambda_{n}^{1 / 2} \lambda_{m}^{1 / 2} a_{j}^{\lambda_{n}+\lambda_{m}}\right) \\
& \leq e \alpha_{n}^{-1} \alpha_{m}^{-1}\left(\frac{1}{4} \alpha_{n} \alpha_{m} \beta_{n m}^{1 / 2}+\frac{\alpha_{n}^{2}}{\lambda_{n}} \lambda_{n}^{1 / 2} \lambda_{m}^{1 / 2} a_{n}^{\lambda_{n}+\lambda_{m}}+\sum_{j \geq n+1} \frac{\alpha_{j}^{2}}{\lambda_{j}} \lambda_{n}^{1 / 2} \lambda_{m}^{1 / 2} a_{j}^{\lambda_{n}+\lambda_{m}}\right) \\
& \leq e \alpha_{n}^{-1} \alpha_{m}^{-1}\left(\frac{1}{4} \alpha_{n} \alpha_{m} \beta_{n m}^{1 / 2}+\frac{\alpha_{n}^{2} \lambda_{m}^{1 / 2}}{\lambda_{n}^{1 / 2}}+\sum_{j \geq n+1} \frac{\alpha_{j}^{2} \lambda_{n}^{1 / 2} \lambda_{m}^{1 / 2}}{\lambda_{j}}\right) \\
& \leq e \alpha_{n}^{-1} \alpha_{m}^{-1}\left(\frac{1}{4} \alpha_{n} \alpha_{m} \beta_{n m}^{1 / 2}+\frac{1}{2} \alpha_{m} \alpha_{n} \beta_{m n}^{1 / 2}+\sum_{j \geq n+1} \frac{1}{2^{j+2-n}} \alpha_{m} \alpha_{n} \beta_{m n}^{1 / 2}\right) \\
& \leq \frac{e}{4} \beta_{n m}^{1 / 2}+\frac{e}{2} \beta_{n m}^{1 / 2}+\frac{e}{4} \beta_{n m}^{1 / 2}=e \beta_{n m}^{1 / 2} .
\end{aligned}
$$

Therefore

$$
\sum_{n \neq m}\left|\left\langle f_{n}, f_{m}\right\rangle_{L^{2}(\mu)}\right|^{2}=2 \sum_{n>m}\left\langle f_{n}, f_{m}\right\rangle_{L^{2}(\mu)}^{2} \leq e \sum_{n, m} \beta_{n m}<\frac{e}{4} .
$$

Consider the Gramian $\Gamma=\left\{\left\langle f_{n}, f_{m}\right\rangle\right\}_{n, m=1}^{\infty}$ of the sequence $\left(f_{n}\right)$. If we define $\Gamma_{0}=\Gamma-I$, then

$$
\begin{aligned}
\left\|\Gamma_{0}\right\|_{\mathcal{S}_{2}}^{2} & =\sum_{n}\left\|\Gamma_{0} e_{n}\right\|_{\ell^{2}}^{2}=\sum_{n, m}\left|\left\langle\Gamma_{0} e_{n}, e_{m}\right\rangle_{\ell^{2}}\right|^{2} \\
& =\sum_{n \neq m}\left|\left\langle\Gamma e_{n}, e_{m}\right\rangle_{\ell^{2}}\right|^{2}=\sum_{n \neq m}\left|\left\langle f_{n}, f_{m}\right\rangle_{L^{2}(\mu)}\right|^{2}<\frac{e}{4}
\end{aligned}
$$

by (7.10). Therefore $\left\|\Gamma_{0}\right\| \leq\left\|\Gamma_{0}\right\|_{\mathcal{S}_{2}}<\sqrt{e} / 2$, whence $\Gamma$ is invertible. This implies by Lemma 2.1 (i) that $\left(f_{n}\right)$ is a Riesz sequence in $L^{2}(\mu)$.

By (7.9) and the choice of $\alpha_{n}$, the sequence $\left(\left\|i_{\mu}^{2} g_{n}\right\|\right)$ is in $\ell_{q}$ but not in $\ell_{r}$. Corollary 2.3 (i) implies then that $i_{\mu}^{2}$ is in $\mathcal{S}_{q}$ but not in $\mathcal{S}_{r}$, as desired.

\section{FinAl REMARKS}

It is often the case when dealing with Müntz spaces that results that are valid for lacunary sequences can be extended, albeit sometimes after significant work, to the quasilacunary case. We have already seen such a situation in Section 6, where the proof of Theorem 6.1 would be actually simpler if one assumes $\Lambda$ to be lacunary. In particular, the continuity of the embedding $i_{\mu}^{1}$ for sublinear measures is shown in [6] for quasilacunary sequences. However, a similar result is not yet proved for $i_{\mu}^{2}$; at least the proof of our Theorem 4.3 does not seem to extend easily to quasilacunary sequences. It is an open problem to provide such an extension.

Another interesting open question is the possible extension of Corollary 5.2 to the range $2<p<\infty$; in particular, is it true in that case that if $\Lambda$ is lacunary, then any sublinear measure is $\Lambda_{p}$-embedding? 
Finally, let us note that an important application of embedding theorems is the study of boundedness properties of composition and multiplication operators with domain Müntz spaces (see [1, 2, 6]). We will give such applications in a forthcoming paper.

\section{REFERENCES}

[1] I. Al Alam. Géometrie des espaces de Müntz et opérateurs de composition à poids. $\mathrm{PhD}$ thesis, Université Lille 1, 2008.

[2] I. Al Alam. Essential norms of weighted composition operators on Müntz spaces, J. Math. Anal. Appl., 358(2), 2009. MR2532505 (2010f:47046)

[3] J. Bergh and J. Löfström. Interpolation Spaces: An Introduction. Springer-Verlag, New York, 1976. MR0482275 (58:2349)

[4] P. Borwein and T. Erdelyi. Polynomials and Polynomial Inequalities, volume 161 of Graduate Texts in Mathematics. Springer-Verlag, New York, 1995. MR1367960 (97e:41001)

[5] Th. J. Bromwich. An Introduction to the Theory of Infinite Series. Macmillan, London, 1908.

[6] I. Chalendar, E. Fricain, and Dan Timotin. Embedding theorems for Müntz spaces, Ann. Inst. Fourier (Grenoble), 61 (2011), 2291-2311. MR2976312

[7] Ole Christensen. An Introduction to Frames and Riesz Bases, Applied and Numerical Harmonic Analysis. Birkhäuser, Boston, 2003. MR1946982 (2003k:42001)

[8] J. Diestel, H. Jarchow, and A. Tonge. Absolutely Summing Operators, volume 43 of Cambridge Studies in Advanced Mathematics. Cambridge University Press, New York, 1995. MR 1342297 (96i:46001)

[9] V.I. Gurariy and W. Lusky. Geometry of Müntz Spaces and Related Questions, volume 1870 of Lecture Notes in Mathematics. Springer-Verlag, Berlin, 2005. MR2190706 (2007g:46027)

[10] Charles A. McCarthy. $c_{p}$, Israel Journal of Mathematics, 5 (1967), 249-271. MR.0225140 $(37: 735)$

[11] A. Spalsbury. Perturbations in Müntz's theorem, J. Approx. Theory, 150(1) (2008), 48-68. MR2381528 (2009e:41038)

Abdus Salam School of Mathematical Sciences, New Muslim Town, Lahore, 54600, PAKISTAN

E-mail address: waleed_math@hotmail.com

Institute of Mathematics of the Romanian Academy, Calea Griviței 21, Bucharest, ROMANIA

E-mail address: Dan.Timotin@imar.ro 